\title{
Altered development of dopaminergic neurons differentiated from stem cells from human exfoliated deciduous teeth of a patient with Down syndrome
}

Thanh Thi Mai Pham ${ }^{1}$, Hiroki Kato ${ }^{1 *} \mathbb{D}$, Haruyoshi Yamaza ${ }^{1}$, Keiji Masuda ${ }^{1}$, Yuta Hirofuji ${ }^{1}$, Hiroshi Sato ${ }^{1}$,

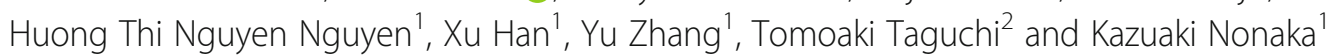

\begin{abstract}
Background: Down syndrome (DS) is a common developmental disorder resulting from the presence of an additional copy of chromosome 21. Abnormalities in dopamine signaling are suggested to be involved in cognitive dysfunction, one of the symptoms of DS, but the pathophysiological mechanism has not been fully elucidated at the cellular level. Stem cells from human exfoliated deciduous teeth (SHED) can be prepared from the dental pulp of primary teeth. Importantly, SHED can be collected noninvasively, have multipotency, and differentiate into dopaminergic neurons (DN). Therefore, we examined dopamine signaling in DS at the cellular level by isolating SHED from a patient with DS, differentiating the cells into DN, and examining development and function of DN.

Methods: Here, SHED were prepared from a normal participant (Ctrl-SHED) and a patient with DS (DS-SHED). Initial experiments were performed to confirm the morphological, chromosomal, and stem cell characteristics of both SHED populations. Next, Ctrl-SHED and DS-SHED were differentiated into DN and morphological analysis of DN was examined by immunostaining. Functional analysis of DN was performed by measuring extracellular dopamine levels under basal and glutamate-stimulated conditions. In addition, expression of molecules involved in dopamine homeostasis was examined by quantitative real-time polymerase chain reaction and immunostaining. Statistical analysis was performed using two-tailed Student's t-tests.

Results: Compared with Ctrl-SHED, DS-SHED showed decreased expression of nestin, a neural stem-cell marker. Further, DS-SHED differentiated into DN (DS-DN) exhibiting decreased neurite outgrowth and branching compared with Ctrl-DN. In addition, DS-DN dopamine secretion was lower than Ctrl-DN dopamine secretion. Moreover, aberrant expression of molecules involved in dopaminergic homeostasis was observed in DS-DN.

Conclusions: Our results suggest that there was developmental abnormality and DN malfunction in the DS-SHED donor in this study. In the future, to clarify the detailed mechanism of dopamine-signal abnormality due to DN developmental and functional abnormalities in DS, it is necessary to increase the number of patients for analysis. Non-invasively harvested SHED may be very useful in the analysis of DS pathology.
\end{abstract}

Keywords: Down syndrome, Dopamine, Human exfoliated deciduous teeth, SHED, Stem cells, Dopaminergic neurons, Differentiation, Dopamine secretion

\footnotetext{
* Correspondence: kato@dent.kyushu-u.ac.jp

${ }^{1}$ Section of Oral Medicine for Child, Division of Oral Health, Growth \&

Development, Faculty of Dental Science, Kyushu University, Maidashi 3-1-1,

Higashi-Ku, Fukuoka 812-8582, Japan

Full list of author information is available at the end of the article
}

(c) The Author(s). 2018 Open Access This article is distributed under the terms of the Creative Commons Attribution 4.0 International License (http://creativecommons.org/licenses/by/4.0/), which permits unrestricted use, distribution, and reproduction in any medium, provided you give appropriate credit to the original author(s) and the source, provide a link to the Creative Commons license, and indicate if changes were made. The Creative Commons Public Domain Dedication waiver (http://creativecommons.org/publicdomain/zero/1.0/) applies to the data made available in this article, unless otherwise stated. 


\section{Background}

Down syndrome (DS) is caused by an extra copy of chromosome 21 and is one of the most common developmental disorders. Reported symptoms of DS include impairment of cognitive functions, such as learning, memory, language, and executive function [1-4]. Dopamine (DA) is an important neurotransmitter in the regulation of cognitive function. It has been suggested that disturbance of the DA signaling system causes the cognitive impairments observed in DS [5-7].

The amount of DA in the brain and cerebrospinal fluid (CSF) of patients with DS has been reported to be both higher and lower than that in healthy people [8, 9], suggesting that a disturbance in DA homeostasis is implicated in DS. It has also been reported that there is reduced expression of DA receptors D1R and D2R in the brains of patients with DS [10]. As observed in patients with DS, varied amounts of DA have also been reported in mouse models of DS $[6,7,11,12]$, suggesting that variable DA levels are associated with abnormal brain development. However, the role of the DA signaling system in DS pathology has yet to be analyzed at a cellular level.

Stem cells from human exfoliated deciduous teeth (SHED) can be acquired noninvasively and used for research [13-15]. Thus, using SHED, consent to participate in research may be obtained more readily from the parents of young patients. SHED can be differentiated into dopaminergic neurons (DN) and used for the treatment of a parkinsonian rat model $[16,17]$. The authors have also previously used SHED derived from a patient with Rett syndrome to elucidate the relationship between abnormal DN development and decreased mitochondrial function in vitro [18]. Therefore, SHED are a valuable source of stem cells for DN transplantation and for in vitro disease models.

The aim of this study was to elucidate a relationship between DS and abnormal DN development and function. Here, SHED were prepared from a normal participant and a patient with DS and were then used to examine DS pathology on a cellular level. Our results demonstrate the utility of SHED as a disease model for DS.

\section{Methods}

\section{Isolation and preparation of SHED}

Human exfoliated deciduous teeth were provided by Pediatric Dentistry and Special Need Dentistry at Kyushu University Hospital in Japan. After informed parental consent was obtained, deciduous teeth were collected from a normal participant and a patient with DS at 6 and 14 years of age, respectively. The isolation procedure was completed as previously described [15]. Briefly, the pulp tissue was subjected to an enzymatic dissociation in $3 \mathrm{mg} / \mathrm{mL}$ collagenase I (Washington, NJ, USA) and $4 \mathrm{mg} / \mathrm{mL}$ dispase II (Wako, Osaka, Japan) for $1 \mathrm{~h}$, and then maintained at $37{ }^{\circ} \mathrm{C}$ in a humidified $5 \% \mathrm{CO}_{2}$ incubator in the Alpha modification of Eagle's Minimal Essential Medium ( $\alpha$-MEM; Sigma-Aldrich, MO, USA) containing 15\% fetal bovine serum (Sigma-Aldrich), $100 \mu \mathrm{M}$ L-ascorbic acid 2-phosphate (Wako), 2 mM L-glutamine (Life Technologies, NY, USA), $250 \mu \mathrm{g} / \mathrm{mL}$ Fungizone (Life Technologies), $100 \mathrm{U} / \mathrm{mL}$ penicillin (Life Technologies), and $100 \mu \mathrm{g} / \mathrm{mL}$ streptomycin (Life Technologies). Cells of not more than 10 passages were used, but Ctrl-SHED and DS-SHED were not always of the same passage.

\section{Fluorescence in situ hybridization}

SHED were treated with $75 \mathrm{mM} \mathrm{KCl}$ for $40 \mathrm{~min}$ and then fixed with 3:1 ethanol:acetic acid $(v / v)$. Fluorescence in situ hybridization (FISH) of chromosome 21 was performed with a chromosome 21 control probe labeling the BAC probe, followed by the standard procedure with green 5-Fluorescein dUTP (CHR21-10-GR; Empire Genomics, NY, USA). Hybridization was performed by denaturing the slides in $70 \%$ formamide/ $2 \times$ standard saline citrate, dehydrating the slides with serial ethanol washes, and applying the probe to the slides. Post-hybridization, the slides were washed and stained with $0.1 \mu \mathrm{g} / \mathrm{mL} \mathrm{4',6-diamidino-2-phe-}$ nylindole (DAPI; Dojindo, Kumamoto, Japan) to identify nuclei. Fluorescence images were taken with a Zeiss Axio Imager M2 microscope (Zeiss, Oberkochen, Germany) equipped with ApoTome2 (Zeiss).

\section{Western blotting}

Whole-cell lysates were extracted with lysis buffer $(62.5 \mathrm{mM}$ Tris- $\mathrm{HCl}$ pH 6.8, 2\% SDS, 5\% $\beta$-mercaptoethanol, and 10\% glycerol), and the protein concentration was measured using Bradford ULTRA (Novexin, Cambridge, UK). A total of $5 \mu \mathrm{g}$ of protein was separated by SDS-PAGE and transferred to a polyvinylidene difluoride membrane. After blocking with $5 \%$ non-fat milk for $30 \mathrm{~min}$, the membrane was incubated overnight at $4{ }^{\circ} \mathrm{C}$ with anti-nestin (1:1000; Millipore, CA, USA) and anti-HSP90 (1:1000; Santa Cruz Biotechnology, CA, USA) antibodies. Membranes were washed and incubated with HRP-conjugated secondary antibody (1:5000; Santa Cruz Biotechnology) for $1 \mathrm{~h}$ at room temperature and visualized with ECL prime (GE Healthcare, Buckinghamshire, UK). The chemiluminescent signals were detected and quantified using LAS-1000 pro (Fuji Film, Tokyo, Japan) with Image Gauge software (Fuji Film). HSP90 was used as an internal control. To normalize the nestin expression, the chemiluminescent signal of nestin was divided by the chemiluminescent signal of HSP90.

\section{DN differentiation}

DN differentiation was induced as previously described with minor modifications (brain derived neurotrophic factor [BDNF] was excluded in the second step) [16]. In 
the first step, $1.5 \times 10^{5}$ SHED were plated onto a 6-well culture plate or glass coverslips coated with $0.01 \%$ poly-Llysine (Sigma-Aldrich), in the same culture medium as described above. They were incubated overnight at $37^{\circ} \mathrm{C}$ in the presence of $5 \% \mathrm{CO}_{2}$, and were then cultured in serum-free Dulbecco's Modified Eagle's Medium (DMEM, Sigma-Aldrich) supplemented with $20 \mathrm{ng} / \mathrm{mL}$ epidermal growth factor (Sigma-Aldrich), $20 \mathrm{ng} / \mathrm{mL}$ basic fibroblast growth factor (Peprotech, NJ, USA), and 1\% N2 supplement (Life Technologies) for 2 days at $37{ }^{\circ} \mathrm{C}$, in the presence of $5 \% \mathrm{CO}_{2}$. In the second step, DMEM was replaced with neurobasal medium (Life Technologies) supplemented with $2 \%$ B27 supplement (Life Technologies), $1 \mathrm{mM}$ dibutyryladenosine 3,5-cyclic monophosphate (Sigma-Aldrich), $0.5 \mathrm{mM}$ 3-isobutyl-1-methylxanthine (Sigma-Aldrich), and $200 \mu \mathrm{M}$ ascorbic acid (Nacalai Tesque, Kyoto, Japan), and cells were incubated for 5 days, at $37^{\circ} \mathrm{C}$, in the presence of $5 \% \mathrm{CO}_{2}$.

\section{Immunocytochemistry}

The cells cultured on coverslips were fixed with $4 \%$ paraformaldehyde in $0.1 \mathrm{M}$ phosphate buffer ( $\mathrm{pH} 7.4$ ) for $10 \mathrm{~min}$. The cells were permeabilized with $0.1 \%$ TritonX-100 for $5 \mathrm{~min}$, then blocked with $2 \%$ bovine serum albumin (BSA; Wako) in PBS for $20 \mathrm{~min}$ at room temperature. Next, cells were stained with primary antibodies against STRO-1 (1:100; Millipore), nestin (1:250; Millipore), $\beta$-tubulin III (1:250; Sigma-Aldrich), tyrosine hydroxylase (TH; 1:100; Millipore), N-methyl-d-aspartate receptor subunit 1 (NMDAR1; 1:100; Millipore), and DA (1:200; Abcam) for 90 min. Following this, cells were incubated with Alexa Fluor secondary antibodies (1:500; Life Technologies) for $1 \mathrm{~h}$ at room temperature in the dark. The cells were counterstained with $0.1 \mu \mathrm{g} / \mathrm{mL}$ DAPI (Dojindo) for $5 \mathrm{~min}$, and then mounted with ProLong diamond (Life Technologies). The fluorescence images were taken with Nikon $\mathrm{C} 2$ confocal microscope (Nikon, Tokyo, Japan) in Fig. 1c and 3a, with Zeiss LSM700 confocal scanning microscope (Zeiss) in Fig. 2a and d, with Zeiss Axio Imager M2 microscope (Zeiss) equipped with ApoTome2 (Zeiss) in Fig. 4c and d.

\section{Morphological analysis of DN}

For morphological analysis, the TH-immunostained images were analyzed with MetaMorph software (Molecular Devices, CA, USA). A morphological analysis of DN was performed as previously described [19]. Briefly, neurite length and number of branches were measured from 100 $\mathrm{TH}$-positive cells in 20 non-overlapping $\mathrm{TH}$-immunostained images selected randomly from three experiments using the Neurite Outgrowth and Multi-Wavelength Cell Scoring module of MetaMorph software (Molecular Devices). Next, the cells were classified into 4 stages based on neurite length and cell diameter as previously described [19].

\section{Measurement of NMDAR1 puncta in neurite}

To measure the number of NMDAR1 puncta per unit length of neurite, DN were immunostained with antiNMDAR1 and anti-TH antibodies. Thirty neurites that were in focus and clearly observed were chosen from 10 Ctrl-DN and 12 DS-DN that were positive for both NMDAR1 and TH. These cells were randomly chosen from three experiments. The number of NMDAR1 puncta per $25 \mu \mathrm{m}$ of neurite was analyzed.

\section{Extracellular DA measurement}

Extracellular DA was measured using a Dopamine Research ELISA kit (BA E-5300, LDN, Nordhorn, Germany) according to the manufacturer's instructions. SHED were plated at a concentration of $5 \times 10^{5}$ cells per 6 - $\mathrm{cm}$ dish and differentiated into DN. $500 \mu \mathrm{l}$ of culture medium was collected to measure extracellular DA. To measure extracellular DA under glutamate stimulated conditions, the cells were treated with $30 \mu \mathrm{M} \mathrm{L}$-glutamate for $1 \mathrm{~min}$ at $37^{\circ} \mathrm{C}$ before harvesting the medium. Next, the cell culture medium was centrifuged at 20,400 $\mathrm{g}$ for $5 \mathrm{~min}$ at $4{ }^{\circ} \mathrm{C}$ to remove cell debris and immediately stored at $-80{ }^{\circ} \mathrm{C}$ until assayed. Subsequently, total protein was extracted from cells using lysis buffer (62.5 mM Tris- $\mathrm{HCl}$ pH 6.8 supplemented with $2 \%$ SDS, 5\% $\beta$-mercaptoethanol, and 10\% glycerol), and the protein concentration was measured using Bradford ULTRA (Novexin). To normalize the DA amount in each sample, the DA amount was divided by the total protein of that sample.

\section{RNA extraction and quantitative real-time polymerase chain reaction (RT-qPCR)}

Total RNA was extracted from the cells using an RNAeasy Mini Kit (Qiagen, Hilden, Germany). First-strand cDNA was synthesized using a ReverTra Ace qPCR RT Master Mix with gDNA Remover (Toyobo, Osaka, Japan). The sequences of primer sets used in this study were as follows: DAT1: 5'-TGCTGCACAGACACCGTGAG-3' (forward), 5'-AATGGTCCAGGAGCGTGAAGA-3' (reverse); VMA T2: 5'-TGAAGAGAGAGGCAACGTCA-3' (forward), 5'-CGTCTTCCCCACAAACTCAT-3 (reverse); HPRT1: 5'-CCTGGCGTCGTGATTAGTG-3' (forward), 5'-TCCC ATCTCCTTCATCACATC-3' (reverse). Real-time quantitative PCR was performed using GoTaq qPCR Master Mix (Promega, WI, USA) and analyzed with StepOnePlus Real-Time PCR Systems (Life Technologies). The threshold cycle $(\mathrm{Ct})$ value of HPRT1 was subtracted from the $\mathrm{Ct}$ value of the target genes $(\Delta \mathrm{Ct})$. Statistical analysis was performed using the $\Delta \mathrm{Ct}$ values from four experiments. The relative expressions of the target genes are shown as fold changes determined using the $2^{-\Delta \Delta \mathrm{Ct}}$ method.

\section{Statistical analysis}

Values are represented as mean \pm standard error of the mean (SEM) from at least three experiments. Two-tailed 


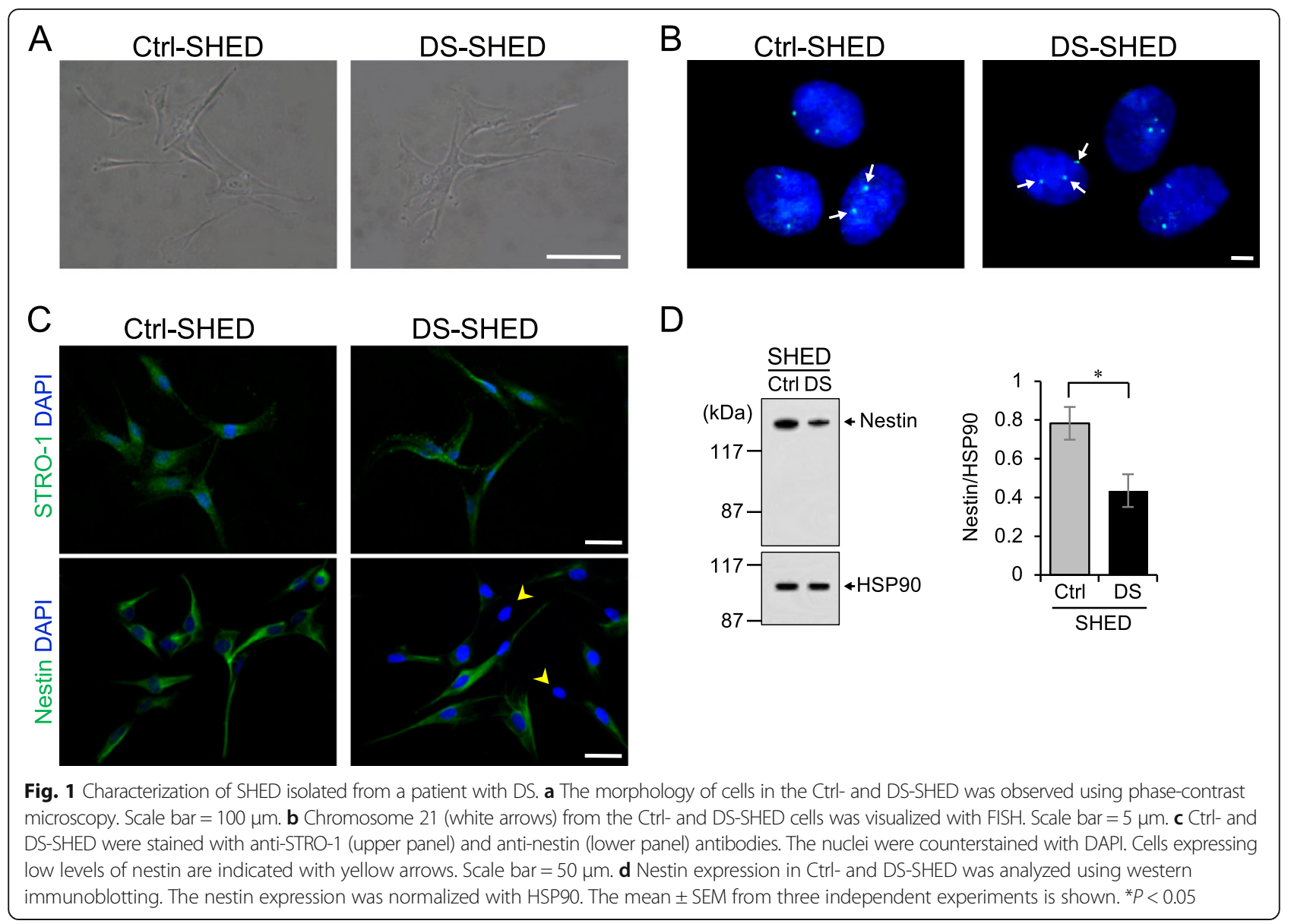

Student's $t$-tests were used to compare the Ctrl and DS groups. Differences were considered significant if $p<$ 0.05. JMP software (SAS Institute, NC, USA) was used for the statistical analysis.

Methods of figure S1 and S2 are described in Additional file 4.

\section{Results}

\section{Characteristics of SHED isolated from a patient with DS}

We isolated SHED from the deciduous teeth of a child with DS (DS-SHED) and a normal participant (Ctrl-SHED). DS-SHED were spindle-shaped and exhibited a fibroblastic cell morphology that was similar to Ctrl-SHED (Fig. 1a). The presence of 3 copies of chromosome 21 in the nuclei of DS-SHED was verified by FISH (Fig. 1b). Next, analysis of cell proliferation showed similar proliferation of DS-SHED and Ctrl-SHED (Additional file 1: Figure S1). SHED have multi-lineage potential and express mesenchymal and neuronal stem cell markers. To examine the stem cell characteristics of DS-SHED, immunofluorescence staining was performed using antibodies against stem cell markers. Both DS-SHED and Ctrl-SHED expressed STRO-1, a mesenchymal stem cell marker (Fig. 1c, upper panel). In contrast, expression of nestin, a neuronal stem cell marker, was reduced in DS-SHED compared to Ctrl-SHED (Fig. 1c; lower panel; yellow arrows denote cells with weak nestin expression). Western blotting (Fig. 1d) and flow cytometry analysis (Additional file 2: Figure S2) were also used to examine nestin expression and showed reduced nestin expression in DS-SHED compared to Ctrl-SHED.

\section{Altered differentiation of DS-SHED into DN}

We differentiated Ctrl-SHED and DS-SHED into DN, and immunostained these with antibodies to the neuronal marker $\beta$-tubulin III and DN marker TH. DN differentiated from DS-SHED (DS-DN) expressed $\beta$-tubulin III and TH (Fig. 2a), but neurite length and branching were reduced compared to DN differentiated from Ctrl-SHED (Ctrl-DN). Quantitative analysis also showed that neurite length and branching were reduced in TH-expressing DS-DN compared to Ctrl-DN (Fig. 2b, c). DN development, evaluated in 4 stages according to cell morphology (Fig. 2d; based on a report by Leach et al.) [19], showed that DS-DN development was reduced compared to Ctrl-DN (Fig. 2e).

\section{Disturbance of DA secretion in DS-DN}

A functional analysis of $\mathrm{DN}$ was performed by examining DA expression and DA secretion. DA expression was 


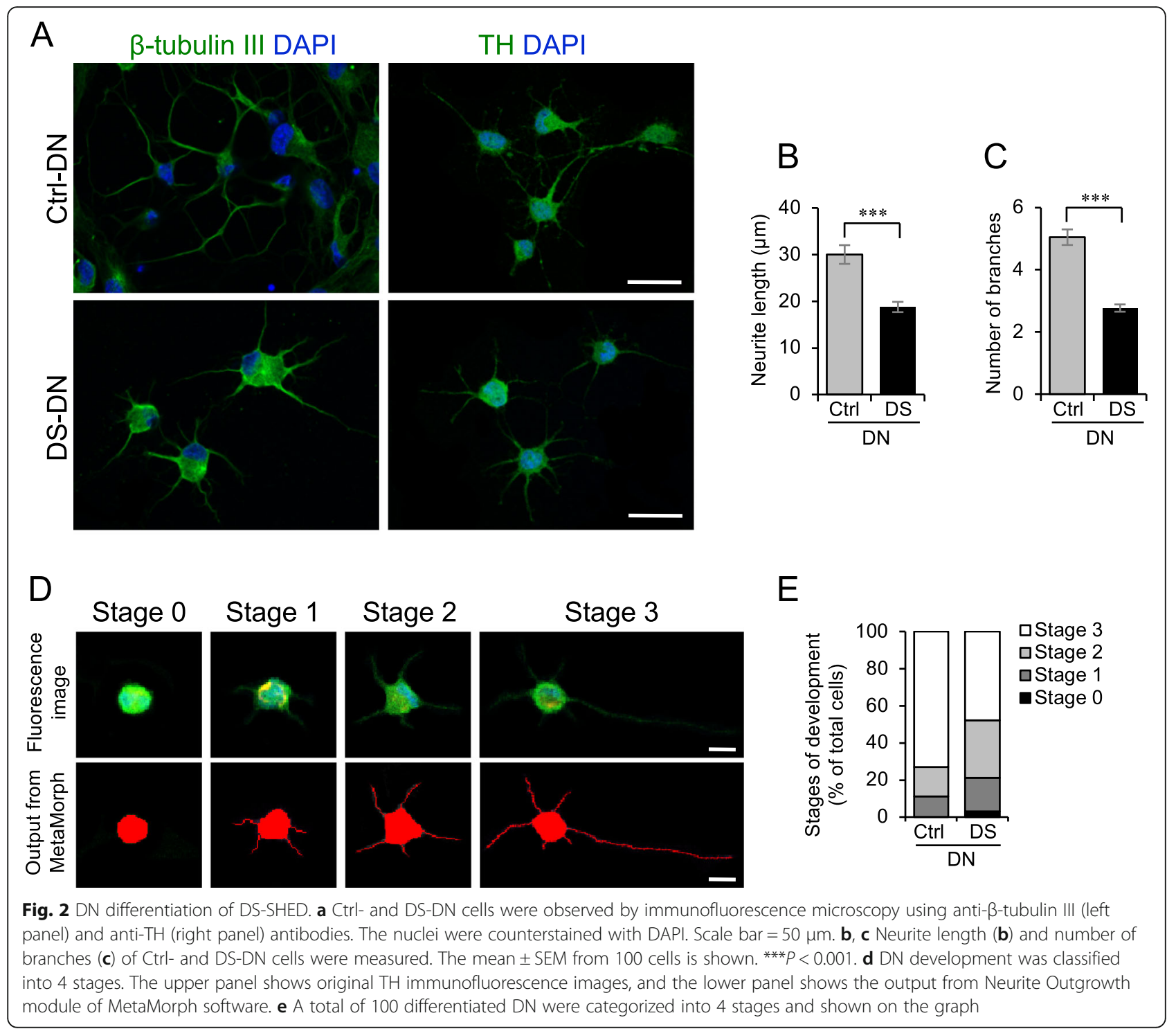

examined by immunostaining cells, which showed that DA expression was present in both Ctrl- and DS-DN (Fig. 3a). Next, DA secretion was examined by measuring extracellular DA and it was observed that extracellular DA of DS-DN was significantly reduced compared with that of Ctrl-DN under basal conditions ( $p=0.046$; Fig. 3b). Although no significant differences between the DS- and Ctrl-DN were observed $(P=0.506)$, extracellular DA of DS-DN was lower than that of Ctrl-DN under glutamate stimulated conditions (Fig. 3c).

\section{Aberrant expression of molecules involved in DA homeostasis in DS-DN}

Possible causes of reduced DA section from DS-DN include the abnormal expression of molecules involved in DA homeostasis, such as dopamine transporter 1 (DAT1) that mediates DA reuptake, vesicular monoamine transporter 2 (VMAT2) that mediates packaging of DA into secretory vesicles, and glutamate receptors. Analysis of DAT1 and VMAT2 mRNA expression showed that DAT1 expression was greater and VMAT2 expression was reduced in DS-DN compared to Ctrl-DN (Fig. 4a, b). Furthermore, expression of NMDAR1, a subunit of glutamate receptor, by immunostaining showed that the number of NMDAR1 puncta per unit length of neurite was reduced in DS-DN compared to Ctrl-DN (Fig. 4c-e).

\section{Discussion}

In this study, various tests on SHED derived from a patient with DS suggest that DN development is reduced and DN function is disturbed in DS. Previous studies have implicated abnormal neuronal cell development in DS based on 


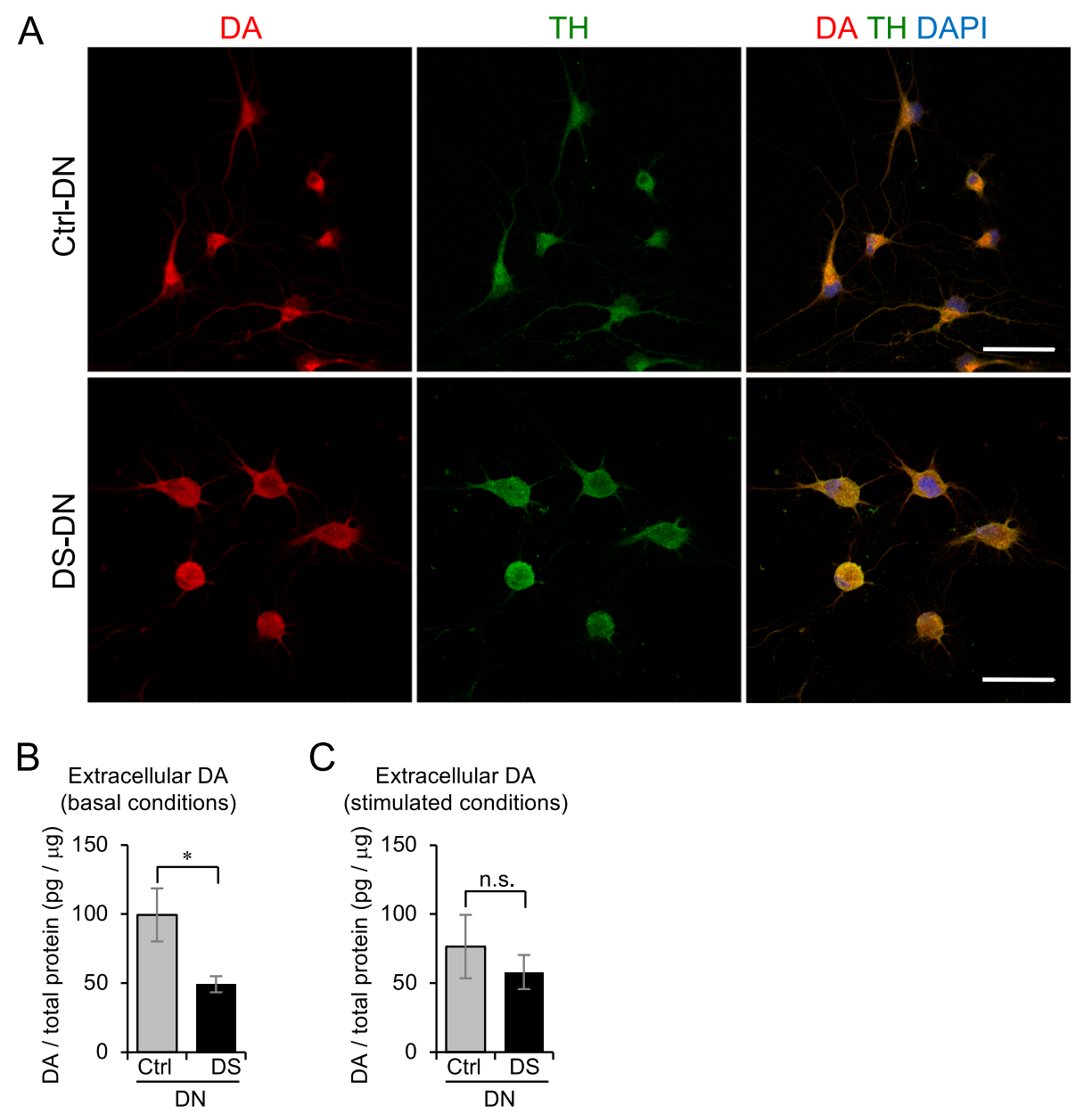

Fig. 3 Altered DA secretion in DS-DN. a DA expression in Ctrl- and DS-DN cells was observed by immunofluorescence microscopy. Ctrl- and DSDN cells were stained with anti-DA and anti-TH antibodies, and fluorescence images were captured using the same acquisition settings. Nuclei were counterstained with DAPI; merged images are shown in the right panels. Scale bar $=50 \mu \mathrm{m}$. b, c Extracellular DA amount under basal conditions $\mathbf{b}$ and glutamate-stimulated conditions $\mathbf{c}$ were measured by ELISA. The DA amount was normalized with total protein extracted from each cell. Graphs show the mean \pm SEM from four experiments. ${ }^{*} P<0.05$; n.S., not significant

the examination of induced pluripotent stem cells (iPSCs) and neurospheres derived from the fetal brains of DS patients [20, 21]; however, these studies did not involve the differentiation of stem cells into a specific type of neuronal cell. Thus, the present study focused on DN and is the first to elucidate a relationship between DS and abnormal DN development and function.

Compared to Ctrl-SHED, the expression of the neuronal stem cell marker nestin was reduced in DS-SHED. It has been reported that when iPSCs from a patient with DS are induced to become neural progenitor cells (NPCs), nestin expression, neuronal cell differentiation capacity, neurite length, and synapse formation are reduced compared to controls [20]. In these NPCs, glia markers as well as the differentiation into glia were enhanced over the differentiation into neurons [20]. Although glia were not examined in the present study, because nestin expression was reduced in SHED derived from the patient with DS, it is possible that differentiation into glia was also enhanced, though further investigation is required to confirm this.

A reduction in DA secretion was observed from DS-DN compared to Ctrl-DN. Expression of DAT1, which mediates DA reuptake, was increased and expression of VMAT2, which is involved in packaging DA into secretory vesicles, was decreased in DS-DN compared to Ctrl-DN. An increase in DA reuptake and reduction of DA packaging into secretory vesicles may have led to the observed reduction in extracellular DA of DS-DN. Furthermore, though no significant differences between the DS- and Ctrl-DN were observed, extracellular DA of DS-DN was reduced under glutamate stimulated conditions. The amount of NMDAR1 in neurites was reduced in DS-DN, which could explain the observed reduction in extracellular DA under glutamate 


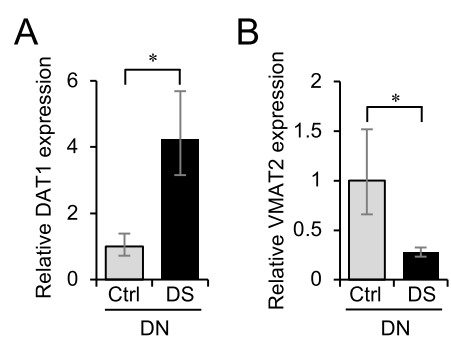

C
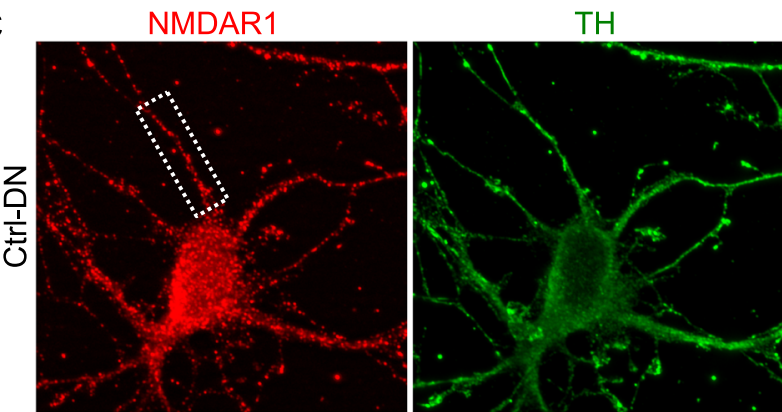

NMDAR1 TH DAPI
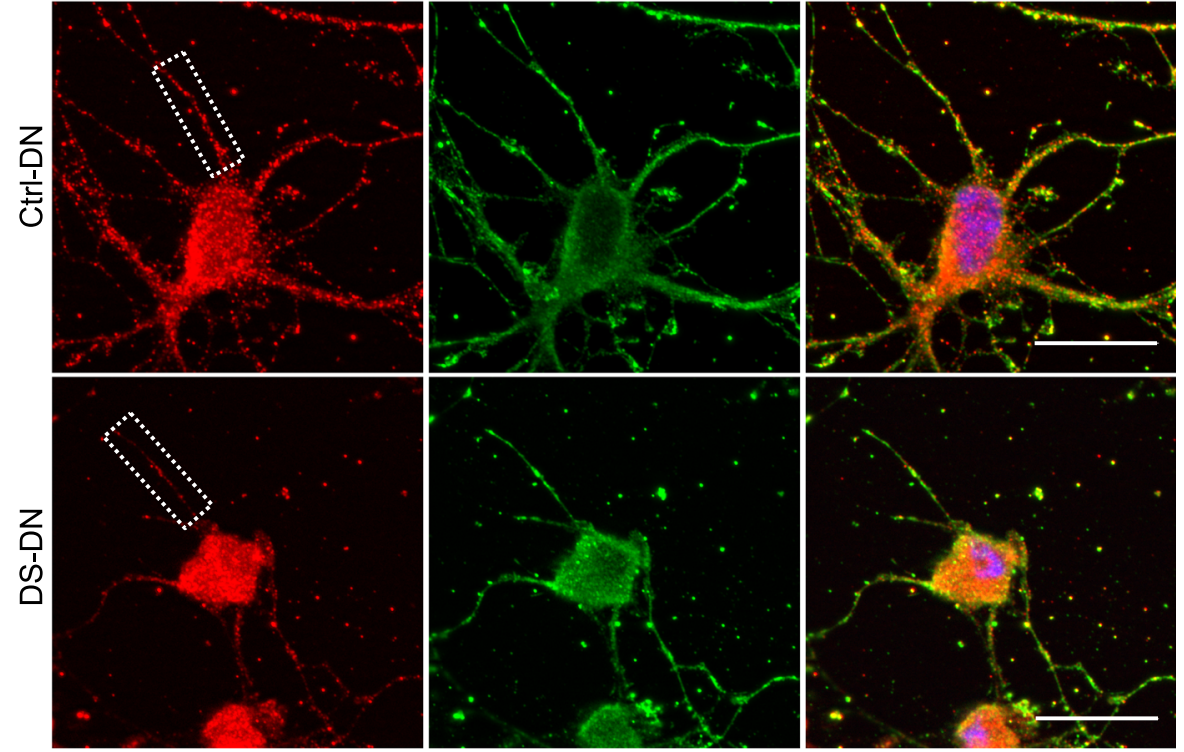

D

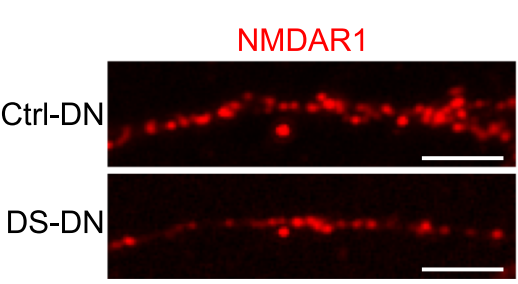

$\mathrm{E}$
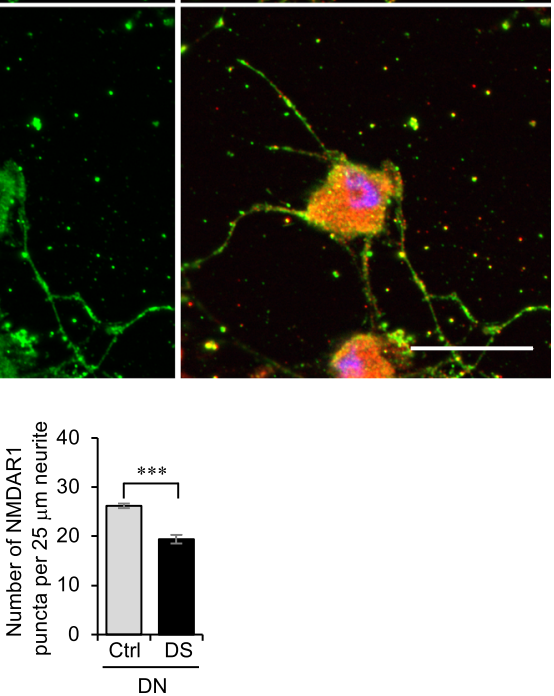

Fig. 4 Aberrant expression of molecules involved in DA homeostasis in DS-DN. $\mathbf{a}$, b The mRNA expression of DAT1 (a) and VMAT2 (b) was measured by RT-qPCR. The relative expression of each gene was calculated with the $2^{-\Delta \Delta C t}$ method. Graphs show the mean \pm SEM from four experiments. ${ }^{*} P<0.05$. c-e NMDAR1 and TH in Ctrl- and DS-DN cells were observed by immunofluorescence microscopy (c). Cells were counterstained with DAPI. Scale bar $=25 \mu \mathrm{m}$. Details of the boxed region in (c) are shown $(\mathbf{d})$. Scale bar $=5 \mu \mathrm{m}$. The number of NMDAR1 puncta per $25 \mu \mathrm{m}$ of neurite was counted (e). Graph shows the mean \pm SEM of NMDAR1 puncta from 30 neurites. ${ }^{* * *} P<0.001$

stimulated conditions. In addition, DAT1 is important for basal extracellular levels of DA [22] and the dramatic change observed in DAT1 could have caused the cause of the greater difference in DA under basal conditions compared to glutamate stimulated conditions.

DS is caused by an extra copy of chromosome 21 and overexpression of the DYRK1a and DSCR1 genes encoded by chromosome 21 are considered to have particularly important roles in the manifestation of DS symptoms [23, 24]. Overexpression of DYRK1a and DSCR1 in mouse brains is reported to delay differentiation of neuronal precursor cells, causing reduced neuronal development [25]. In our study, neurite length and branching as well as development were reduced in DN derived from DS-SHED. We anticipate that DYRK1 $a$ and DSCR1 are involved in this reduced DN development. Further investigation into the expression of these genes and the effects of inhibitors [26, 27] and siRNA in SHED and DN from DS patients are required.

This study used SHED to investigate DS. The stem cell potential of mesenchymal stem cells is reported to change with repeated passages [28]. The present study used SHED of no more than 10 passages. There was no significant difference in cell proliferation and nestin expression between SHED of fewer passages (6 passages) and 10 passages 
(Additional file 1: Figure S1 and Additional file 2: Figure S2); for this reason, passage-related differences were considered to have a minimal effect on our data. Nevertheless, to increase the utility of the SHED disease model, it will be necessary to determine how many passages of SHED can be used by performing repeated passages and obtaining an accurate understanding of its effect on stem cell marker expression and cell proliferation capacity.

In the present study, we differentiated SHED into DN using a 2-step process based on the method by Fujii et al. [16]; they added brain-derived neurotrophic factor (BDNF) to differentiation media in the second step, but we omitted this addition. Aberrant expression of BDNF is reported in DS patients and mouse models of DS [29-31], suggesting a relationship between neuronal development and BDNF in DS. BDNF is secreted extracellularly and mediates neuronal development and survival. BDNF serves autocrine and paracrine functions [32, 33]. If BDNF exhibits abnormal autocrine function in DS, adding BDNF to the media would conceal DS-SHED pathology. For this reason, BDNF was not added to the media in the present study. It will be necessary to examine BDNF and BDNF receptor expression in future studies to elucidate the involvement of BDNF in DN development of DS.

Fujii et al. reported that Ngn2 and Mash1 expression are important as they are activated in the first step of the process that involves differentiation of SHED into early stage DN [16]. In the second step, Fujii et al. speculated that BDNF promotes maturation of early stage DN to DN. When the authors differentiated SHED into DN and performed immunostaining with MAP2 and Tau antibodies, both Ctrl-DN and DS-DN expressed both proteins in whole-cells (Additional file 3: Figure S3). Previous immunostaining studies utilizing mature neuronal cells revealed that the MAP2 antibody stains dendrites, while the Tau antibody stains axons [34, 35]. This suggests that the $\mathrm{DN}$ in this study are still developing and have thus not fully matured. This is a limitation of our study. Further studies involving the addition of BDNF are needed to examine synapse formation and other phenomena in mature DN.

\section{Conclusion}

SHED were prepared from a patient with DS and differentiated into DN, revealing abnormal DN development and function. We predict that this DS patient has abnormal DA signaling, but further investigations, such as analyzing cognitive function and DA levels in this DS patient, are necessary. In the future, it will also be necessary to increase the number of patients for analysis to clarify the disturbance in dopaminergic neurodevelopment implicated in the pathophysiology of DS. SHED, which can be prepared noninvasively, offers an effective disease model for this research.

\section{Additional files}

Additional file 1: Figure S1. Cell proliferation of SHED in different passage. Ctrl- and DS-SHED were cultured for $24 \mathrm{~h}$ and $48 \mathrm{~h}$. The number of cells were counted, and the means \pm SEMs from three experiments are shown in the graph. P6; passage 6. P10; passage 10. n.S., not significant. (PDF $10 \mathrm{~kb}$ )

Additional file 2: Figure S2. Nestin expression in different passages of SHED. Nestin expression in Ctrl- and DS-SHED cells was analyzed with flow cytometry at different passages. P6; passage 6. P10; passage 10. (PDF 186 kb)

Additional file 3: Figure S3. Distribution of Tau and MAP2 in DN derived from SHED in this study. Ctrl- and DS-DN were stained with anti-Tau (1:100; Wako) and anti-MAP2 (1:100; Sigma-Aldrich) antibodies. The cells were counterstained with DAPI. The distribution of Tau and MAP2 was observed with Zeiss Axio Imager M2 microscope (Zeiss) equipped with ApoTome2 (Zeiss). Scale bar $=25 \mu \mathrm{m}$. (PDF $7737 \mathrm{~kb}$ )

Additional file 4: Supplemental methods. Methods for Figure S1 and S2. (DOCX $30 \mathrm{~kb})$

\section{Abbreviations}

DA: Dopamine; DN: dopaminergic neurons; DS: Down Syndrome; NMDAR1: N-methyl-D-aspartate receptor subunit NR1; SHED: stem cells from human exfoliated deciduous teeth; $\mathrm{TH}$ : tyrosine hydroxylase

\section{Acknowledgments}

We thank Drs. Takahiro Kato, Yasunari Sakai and Takayoshi Yamaza (Kyushu University) for their valuable suggestions and technical support. We appreciate the technical assistance provided by the Research Support Center, Research Center for Human Disease Modeling, Kyushu University Graduate School of Medical Sciences.

\section{Funding}

This work was supported by JSPS KAKENHI Grant Numbers JP25670877 and JP16K15839 to KN.

\section{Availability of data and materials}

The data that support the findings of this study are included within the article.

\section{Authors' contributions}

TP, HK: contribution to conception and design the study; acquisition of the data; analysis and interpretation of the data; drafting the manuscript; revising the manuscript; final approval of the version to be published. HY, KM: contribution to conception and design the study; acquisition of the data; revising the manuscript; final approval of the version to be published. $\mathrm{YH}$, $\mathrm{HS}, \mathrm{HN}, \mathrm{XH}, \mathrm{YZ}$ : acquisition of the data; drafting the manuscript; final approval of the version to be published. $T$, KN: contribution to conception and design the study; analysis and interpretation of the data; revising the manuscript; final approval of the version to be published.

\section{Ethics approval and consent to participate}

Experiments using human samples were reviewed and approved by the Kyushu University Institutional Review Board for Human Genome/Gene Research (approval number 678-00) and were conducted in accordance with the Declaration of Helsinki. Written informed consent was obtained from the patient's guardians.

\section{Consent for publication}

Not applicable.

\section{Competing interests}

The authors declare that they have no competing interests.

\section{Publisher's Note}

Springer Nature remains neutral with regard to jurisdictional claims in published maps and institutional affiliations. 


\section{Author details}

${ }^{1}$ Section of Oral Medicine for Child, Division of Oral Health, Growth \& Development, Faculty of Dental Science, Kyushu University, Maidashi 3-1-1, Higashi-Ku, Fukuoka 812-8582, Japan. '2Department of Pediatric Surgery, Reproductive and Developmental Medicine, Graduate School of Medical Sciences, Kyushu University, Maidashi 3-1-1, Higashi-Ku, Fukuoka 812-8582, Japan.

Received: 20 June 2018 Accepted: 24 August 2018

Published online: 31 August 2018

\section{References}

1. Edgin JO, Mason GM, Spano G, Fernandez A, Nadel L. Human and mouse model cognitive phenotypes in Down syndrome: implications for assessment. Prog Brain Res. 2012;197:123-51. https://doi.org/10.1016/B978-0-444-54299-1. 00007-8.

2. Edgin JO. Cognition in Down syndrome: a developmental cognitive neuroscience perspective. Wiley Interdiscip Rev Cogn Sci. 2013;4(3):307-17. https://doi.org/10.1002/wcs.1221.

3. Fidler DJ, Nadel L. Education and children with Down syndrome: neuroscience, development, and intervention. Ment Retard Dev Disabil Res Rev. 2007;13(3):262-71. https://doi.org/10.1002/mrdd.20166.

4. Lott IT, Dierssen M. Cognitive deficits and associated neurological complications in individuals with Down's syndrome. Lancet Neurol. 2010; 9(6):623-33. https://doi.org/10.1016/S1474-4422(10)70112-5

5. Mason GM, Spano G, Edgin J. Symptoms of attention-deficit/hyperactivity disorder in Down syndrome: effects of the dopamine receptor D4 gene. Am J Intellect Dev Disabil. 2015;120(1):58-71. https://doi.org/10.1352/1944-7558120.1.58.

6. Shimohata A, Ishihara K, Hattori S, Miyamoto H, Morishita H, Ornthanalai G et al. Ts1Cje Down syndrome model mice exhibit environmental stimulitriggered locomotor hyperactivity and sociability concurrent with increased flux through central dopamine and serotonin metabolism. Exp Neurol. 2017;293:1-12. https://doi.org/10.1016/j.expneurol.2017.03.009.

7. London J, Rouch C, Bui LC, Assayag E, Souchet B, Daubigney F, et al. Overexpression of the DYRK1A gene (dual-specificity tyrosine phosphorylation-regulated kinase $1 \mathrm{~A}$ ) induces alterations of the serotoninergic and dopaminergic processing in murine brain tissues. Mol Neurobiol. 2018:55(5):3822-31. https://doi.org/10.1007/s12035-017-0591-6.

8. Kay AD, Schapiro MB, Riker AK, Haxby JV, Rapoport SI, Cutler NR. Cerebrospinal fluid monoaminergic metabolites are elevated in adults with Down's syndrome. Ann Neurol. 1987;21(4):408-11. https://doi.org/10.1002/ ana.410210416.

9. Whittle N, Sartori SB, Dierssen M, Lubec G, Singewald N. Fetal Down syndrome brains exhibit aberrant levels of neurotransmitters critical for normal brain development. Pediatrics. 2007;120(6):e1465-71. https://doi.org/ 10.1542/peds.2006-3448

10. Falsafi SK, Dierssen M, Ghafari M, Pollak A, Lubec G. Reduced cortical neurotransmitter receptor complex levels in fetal Down syndrome brain Amino Acids. 2016:48(1):103-16. https://doi.org/10.1007/s00726-015-2062-6.

11. Singer HS, Tiemeyer M, Hedreen JC, Gearhart J, Coyle JT. Morphologic and neurochemical studies of embryonic brain development in murine trisomy 16. Brain Res. 1984;317(2):155-66.

12. Dekker AD, Vermeiren Y, Albac C, Lana-Elola E, Watson-Scales S, Gibbins D, et al. Aging rather than aneuploidy affects monoamine neurotransmitters in brain regions of Down syndrome mouse models. Neurobiol Dis. 2017;105: 235-44. https://doi.org/10.1016/j.nbd.2017.06.007.

13. Miura M, Gronthos S, Zhao M, Lu B, Fisher LW, Robey PG, et al. SHED: stem cells from human exfoliated deciduous teeth. Proc Natl Acad Sci U S A. 2003;100(10):5807-12. https://doi.org/10.1073/pnas.0937635100.

14. Kato $H$, Thi Mai Pham T, Yamaza H, Masuda K, Hirofuji $Y$, Han X, et al. Mitochondria Regulate the Differentiation of Stem Cells from Human Exfoliated Deciduous Teeth. Cell Struct Funct. 2017:42(2):105-16. https://doi.org/10.1247/csf.17012.

15. Kato $H$, Han X, Yamaza $H$, Masuda $K$, Hirofuji $Y$, Sato $H$, et al. Direct effects of mitochondrial dysfunction on poor bone health in Leigh syndrome. Biochem Biophys Res Commun. 2017:493(1):207-12. https://doi.org/10.1016/ j.bbrc.2017.09.045.

16. Fujii $H$, Matsubara $K$, Sakai $K$, Ito M, Ohno K, Ueda M, et al. Dopaminergic differentiation of stem cells from human deciduous teeth and their therapeutic benefits for parkinsonian rats. Brain Res. 1613;2015:59-72. https://doi.org/10.1016/j.brainres.2015.04.001.

17. Wang J, Wang $X$, Sun Z, Wang $X$, Yang $H$, Shi S, et al. Stem cells from human-exfoliated deciduous teeth can differentiate into dopaminergic neuron-like cells. Stem Cells Dev. 2010;19(9):1375-83. https://doi.org/10. 1089/scd.2009.0258

18. Hirofuji S, Hirofuji $Y$, Kato H, Masuda K, Yamaza H, Sato H, et al. Mitochondrial dysfunction in dopaminergic neurons differentiated from exfoliated deciduous tooth-derived pulp stem cells of a child with Rett syndrome. Biochem Biophys Res Commun. 2018;498(4):898-904. https://doi.org/10.1016/j.bbrc.2018.03.077.

19. Leach MK, Naim Yl, Feng ZQ, Gertz CC, Corey JM. Stages of neuronal morphological development in vitro--an automated assay. J Neurosci Methods. 2011;199(2):192-8. https://doi.org/10.1016/j.jneumeth.2011.04.033.

20. Hibaoui Y, Grad I, Letourneau A, Sailani MR, Dahoun S, Santoni FA, et al. Modelling and rescuing neurodevelopmental defect of Down syndrome using induced pluripotent stem cells from monozygotic twins discordant for trisomy 21. EMBO Mol Med. 2014;6(2):259-77. https://doi.org/10.1002/ emmm.201302848.

21. Bahn S, Mimmack M, Ryan M, Caldwell MA, Jauniaux E, Starkey M, et al. Neuronal target genes of the neuron-restrictive silencer factor in neurospheres derived from fetuses with Down's syndrome: a gene expression study. Lancet. 2002;359(9303):310-5. https://doi.org/10.1016/s0140-6736(02)07497-4.

22. Gainetdinov RR, Jones SR, Fumagalli F, Wightman RM, Caron MG. Reevaluation of the role of the dopamine transporter in dopamine system homeostasis. Brain Res Brain Res Rev. 1998:26(2-3):148-53.

23. Guimera J, Casas C, Estivill X, Pritchard M. Human minibrain homologue (MNBH/DYRK1): characterization, alternative splicing, differential tissue expression, and overexpression in Down syndrome. Genomics. 1999;57(3): 407-18. https://doi.org/10.1006/geno.1999.5775.

24. Fuentes JJ, Genesca L, Kingsbury TJ, Cunningham KW, Perez-Riba M, Estivill X et al. DSCR1, overexpressed in Down syndrome, is an inhibitor of calcineurinmediated signaling pathways. Hum Mol Genet. 2000;9(11):1681-90.

25. Kurabayashi N, Sanada K. Increased dosage of DYRK1A and DSCR1 delays neuronal differentiation in neocortical progenitor cells. Genes Dev. 2013; 27(24):2708-21. https://doi.org/10.1101/gad.226381.113.

26. Ogawa Y, Nonaka Y, Goto T, Ohnishi E, Hiramatsu T, Kii I, et al. Development of a novel selective inhibitor of the Down syndrome-related kinase Dyrk1A. Nat Commun. 2010;1:86. https://doi.org/10.1038/ncomms1090.

27. Kim H, Lee KS, Kim AK, Choi M, Choi K, Kang M, et al. A chemical with proven clinical safety rescues down-syndrome-related phenotypes in through DYRK1A inhibition. Dis Model Mech. 2016;9(8):839-48. https://doi. org/10.1242/dmm.025668.

28. Zhuang Y, Li D, Fu J, Shi Q, Lu Y, Ju X. Comparison of biological properties of umbilical cord-derived mesenchymal stem cells from early and late passages: immunomodulatory ability is enhanced in aged cells. Mol Med Rep. 2015;11(1):166-74. https://doi.org/10.3892/mmr.2014.2755.

29. Dogliotti G, Galliera E, Licastro F, Corsi MM. Age-related changes in plasma levels of BDNF in Down syndrome patients. Immun Ageing. 2010;7:2. https://doi.org/10.1186/1742-4933-7-2.

30. Troca-Marin JA, Alves-Sampaio A, Montesinos ML. An increase in basal BDNF provokes hyperactivation of the Akt-mammalian target of rapamycin pathway and deregulation of local dendritic translation in a mouse model of Down's syndrome. J Neurosci. 2011;31(26):9445-55. https://doi.org/10. 1523/JNEUROSCI.0011-11.2011.

31. Parrini M, Ghezzi D, Deidda G, Medrihan L, Castroflorio E, Alberti M, et al. Aerobic exercise and a BDNF-mimetic therapy rescue learning and memory in a mouse model of Down syndrome. Sci Rep. 2017;7(1):16825. https://doi. org/10.1038/s41598-017-17201-8.

32. Cheng $\mathrm{PL}$, Song AH, Wong $\mathrm{YH}$, Wang S, Zhang X, Poo MM. Self-amplifying autocrine actions of BDNF in axon development. Proc Natl Acad Sci U S A. 2011;108(45):18430-5. https://doi.org/10.1073/pnas.1115907108.

33. Wang $L$, Chang $X$, She $L, X u$ D, Huang W, Poo MM. Autocrine action of BDNF on dendrite development of adult-born hippocampal neurons. J Neurosci. 2015;35(22):8384-93. https://doi.org/10.1523/JNEUROSCI.4682-14.2015.

34. Dehmelt $\mathrm{L}$, Halpain S. The MAP2/Tau family of microtubule-associated proteins Genome Biol. 2005;6(1):204. https://doi.org/10.1186/gb-2004-6-1-204

35. Kosik KS, Finch EA. MAP2 and tau segregate into dendritic and axonal domains after the elaboration of morphologically distinct neurites: an immunocytochemical study of cultured rat cerebrum. J Neurosci. 1987;7(10): 3142-53. 